Recepción: 19 / 11 / 2018

Aceptación: 28 / 12 / 2018

Publicación: 20 / 02 / 2019

Ciencias de la salud

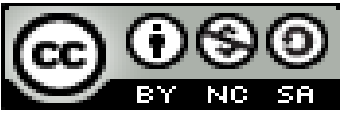

Artículo de revisión

\title{
Consideraciones preventivas para detectar la presencia del papilomavirus
}

\section{Preventive considerations to detect the presence of papillomavirus}

\section{Considerações preventivas para detectar a presença do papilomavírus}

Noralma K. Jaime-Hernandez ${ }^{\text {I }}$ norahernanadez10@hotmail.es

Gerardo A. Granja-Carrión II gerardo_antonio21@hotmail.es

Mario W. Pacheco-Moreira III mariowpacheco@hotmail.com

Yuly S. Mitte-Baque IV mitte52013@hotmail.com

Ana M. Zambrano-Zambrano V ann_mary18@hotmail.com

Jhon A. González-Macas vI jhonandy.gm@hotmail.es

Correspondencia: norahernanadez10@hotmail.es

I. Licenciada en enfermería; Magíster en Emergencias Médicas; Docente de Facultad de Ciencias de la Salud, Carrera Enfermería de la Universidad Estatal del Sur de Manabí; Jipijapa, Ecuador.

III. Médico General en Funciones Hospitalarias en Área de Gineco Obstetricia - Hospital Dr. Gustavo Domínguez; Santo Domingo, Ecuador.

IV. Médico General en Funciones Hospitalarias en Área de Gineco Obstetricia - Hospital Dr. Gustavo Domínguez; Santo Domingo, Ecuador.

v. Médico General de primer nivel de atención en el Distrito 13D10 Jama Pedernales Cojimies. Ecuador.

VI. Médico General; Médico residente del Hospital Básico Limones, Esmeraldas, Ecuador; Médico Residente de la Clínica Profamilia; Quito, Ecuador. 


\section{Resumen.}

El papilomavirus (VPH) es una infección común y la mayoría de las personas contraen en algún momento de su vida. La incidencia máxima de la infección por el VPH se presenta en la adolescencia, poco después de la iniciación de la actividad sexual, y la mayoría de las infecciones se resuelven espontáneamente en un plazo de dos años, sin embargo, aunque no hay una cura para esta infección, las verrugas, lesiones y cambios precancerosos y cancerosos causados por el virus pueden ser tratados. El objetivo de este trabajo investigativo, desarrollado bajo un metodología documental, se centra en realizar una revisión a la literatura científica disponible que principalmente responda a qué es el VPH, cuáles con sus tipos, cómo se detecta y cuáles son las otras alternativas tecnológicas para ello, cuáles son considerados los fatores de riesgo influyentes, cales son las vacunas contra el VPH, cómo puede prevenirse la infección y finalmente cuales con las consideraciones preventivas para la detección, todo ello con la finalidad de proporcionar un material actualizado que defina dichos aspectos. Para ello se escogieron más de dieciséis fuentes físicas, y digitales, ubicadas en bases de datos tales como: Redalyc, Imbiomed, SciELO, Dialnet, Medlineplus, OMS, OPS/PAHO, SCIelo, intramed, entre otras, que fundamentan las ideas aquí planteadas, y que consecuentemente sirven de base para concluir que aún sigue siendo muy importante que mediante programas se brinde la información a la población en general sobre la prevención de la infección por el VPH, de las otras infecciones de transmisión sexual y de comportamientos sexuales sanos, sin embargo, tanto para aquellos países o gobiernos que mediante sus políticas ya han acogido oficialmente alguna prueba para la detección del VPH como para los que aún están en ese proceso, a tales fines, es igualmente recomendable en cuanto a las consideraciones preventivas para detectar el VPH, que tengan en consideración la validación clínica de la prueba, aspectos operacionales y logísticos, además de los costos y beneficios de la prueba.

Palabras claves: Infección; Virus; Factores; Detección; Prueba.

\section{Abstract.}

The papillomavirus (HPV) is a common infection and most people contract at some point in their lives. The maximum incidence of HPV infection occurs in adolescence, shortly after the initiation of sexual activity, and most infections resolve spontaneously within two years, however, although there is no cure for This infection, warts, lesions and precancerous and cancerous changes caused by the virus can be treated. The objective of this research work, developed under a documentary 
Noralma K. Jaime-Hernandez; Gerardo A. Granja-Carrión; Mario W. Pacheco-Moreira; Yuly S. Mitte-Baque; Ana M. Zambrano-Zambrano; Jhon A. González-Macas

methodology, is focused on making a review of the available scientific literature that mainly responds to what HPV is, what its types, how it is detected and what are the other technological alternatives for it., what are considered the influential risk factors, what are the vaccines against HPV, how can infection be prevented and finally what with preventive considerations for detection, all with the purpose of providing updated material that defines these aspects. To this end, more than sixteen physical and digital sources were chosen, located in databases such as: Redalyc, Imbiomed, SciELO, Dialnet, Medlineplus, WHO, PAHO / PAHO, SCIelo, intramed, among others, which support the ideas presented here, and which consequently serve as a basis to conclude that it is still very important that programs provide information to the population in general about the prevention of HPV infection, other sexually transmitted infections and healthy sexual behaviors, However, both for those countries or governments that through their policies have already officially hosted some HPV test and for those who are still in that process, for these purposes, it is also advisable in terms of preventive considerations to detect the HPV. VPH, that take into consideration the clinical validation of the test, operational and logistical aspects, in addition to the costs and benefits of the testing.

Keys words: Infection; Virus; Factors; Detection; Test.

\section{Resumo.}

O papilomavírus (HPV) é uma infecção comum e a maioria das pessoas se contrai em algum momento de suas vidas. A incidência máxima de infecção por HPV ocorre na adolescência, logo após o início da atividade sexual, e a maioria das infecções se resolve espontaneamente dentro de dois anos, embora não haja cura para essa infecção, verrugas, lesões e alterações pré-cancerosas e cancerígenas causadas pelo vírus. Pode ser tratado. O objetivo deste trabalho de pesquisa, desenvolvido sob uma metodologia documental, é focado em fazer uma revisão da literatura científica disponível que responda principalmente ao que é HPV, quais seus tipos, como é detectado e quais são as outras alternativas tecnológicas para o mesmo., quais são os fatores de risco influentes, quais são as vacinas contra o HPV, como a infecção pode ser prevenida e, finalmente, quais as considerações preventivas para detecção, tudo com o objetivo de fornecer material atualizado que defina esses aspectos. Para tanto, foram escolhidas mais de dezesseis fontes físicas e digitais, localizadas em bases de dados como: Redalyc, Imbiomed, SciELO, Dialnet, Medlineplus, OMS, OPAS / OPAS, SCIelo, intramed, entre outras, que apóiam as ideias aqui apresentadas, e que, 
consequentemente, servem como base para concluir que ainda é muito importante que os programas forneçam informações à população em geral sobre a prevenção da infecção pelo HPV, outras infecções sexualmente transmissíveis e comportamentos sexuais saudáveis, no entanto, tanto para países como para governos que suas políticas já realizaram oficialmente alguns testes de HPV e, para aqueles que ainda estão nesse processo, para esses fins, também é aconselhável em termos de considerações preventivas para detectar o HPV. VPH, que leva em consideração a validação clínica do teste, aspectos operacionais e logísticos, além dos custos e benefícios do teste.

Palavras chaves: Infecção Vírus; Fatores Detecção; Teste.

\section{Introducción.}

"Los papilomavirus cohabitan con los humanos desde hace miles de años sufriendo pocos cambios en su composición genómica” (Silva, Waliszewski, Lozoya, \& Carrillo, 2013)

El papilomavirus (Virus de Papiloma Humano / VPH) es una infección que afecta a hombres y mujeres y mundialmente viene siendo la infección de transmisión sexual (ITS) más frecuente. A pesar de que el hombre juega un papel importante en la transmisión de este virus, al estar catalogado como vector sigiloso de este microorganismo, sólo 1\% de ellos experimenta algún signo o síntoma clínico, generalmente asociados a lesiones tipo verrugas (a nivel ano-genitales) y a neoplasias intraepiteliales de pene y ano; ahora, en el caso de las mujeres es todo un contraste, pues esta misma infección, en el 99,7\% de los casos, fundamentalmente viene siendo el agente causal del cáncer cérvico-uterino ( $\mathrm{CCU})$; contexto este por el que evidentemente en el caso masculino se le asume como un problema menor y de escasa relevancia. (Silva et al.; 2013)

En la actualidad, se encuentran en el mercado cerca de cien pruebas comerciales para la detección de VPH y, a pesar que se trata de técnicas estandarizadas, aquellas que no son reproducibles, que no han sido validadas o que carecen de pruebas de fiabilidad y precisión, no se deben utilizar en la gestión clínica ni en programas poblacionales. Por lo tanto, es importante que las pruebas de VPH tengan la máxima sensibilidad clínica sin comprometer la especificidad. (PAHO; 2016; p. 9)

Gonzalez \& Sánchez (2009) citados en la obra de (Rivera et al.; 2018), han sostenido la idea de que: 
Noralma K. Jaime-Hernandez; Gerardo A. Granja-Carrión; Mario W. Pacheco-Moreira; Yuly S. Mitte-Baque; Ana M. Zambrano-Zambrano; Jhon A. González-Macas

De acuerdo con los pocos estudios realizados en América del Sur, además del VPH 16 y el VPH 18, el VPH 58 es otro de los genotipos de alto riesgo encontrados con mayor frecuencia en la región. Este último tipo de VPH ha sido detectado en el centro y norte de Brasil, Argentina, Colombia y Ecuador. (Rivera et al.; 2018)

Seguidamente, los últimos indican que:

En los últimos veinte años se han realizado pocos estudios sobre la epidemiología del VPH en Ecuador. La mayor parte de los estudios efectuados se basan en variados métodos de detección y tipificación de VPH, existiendo múltiples discrepancias en cuanto a la prevalencia de los genotipos de VPH de mayor circulación. Investigaciones recientes muestran que el VPH 16 presenta una alta frecuencia en la población femenina, seguido por el VPH 58, lo cual constituye una novedad para Ecuador. (Rivera et al.; 2018)

El objetivo de este trabajo investigativo se centra en realizar una revisión a la literatura científica disponible que principalmente responda a qué es el VPH, cuáles con sus tipos, cómo se detecta y cuáles son las otras alternativas tecnológicas para ello, cuáles son considerados los fatores de riesgo influyentes, cales son las vacunas contra el VPH, cómo puede prevenirse la infección y finalmente cuales con las consideraciones preventivas para la detección, todo ello con la finalidad de proporcionar un material actualizado que defina dichos aspectos.

\section{Materiales y Métodos}

Esta revisión se ha desarrollado en el marco de una investigación documental. A tales fines se recurre a la utilización de diferentes materiales y fuentes bibliográficas oficiales y formales, tales como libros, revistas científicas especializadas, boletines informativos, folletos, tesis, datos estadísticos, a los que se accedió de manera física y digital mediante el uso de diversas bases de datos, entre las que figuran las de: Redalyc, Imbiomed, SciELO, Dialnet, Medlineplus, OMS, OPS/PAHO, SCIelo, intramed, entre otras.

El desarrollo de dicha investigación se llevó a cabo mediante la observación, orden, clasificación, resumen, análisis deductivo y discusión de todo material, el cual fue seleccionado en base a criterios de temática, idioma español e inglés, relevancia y en todo momento procurando una vigencia en los 
últimos 6 años; siendo escogidos poco más de doce temas que en conjunto permitieron fundamentar científicamente las bases teóricas y científicas de la argumentación plasmada aquí en equipo, que en definitiva facilitó la construcción de un solo criterio el cual se ha expuesto entre los resultados y conclusiones obtenidas.

Ahora bien, es pertinente conceptualizar lo que se entiende por investigación documental, y para ello se hace referencia al aporte de Alfonso (1995) quien citado en Morales (2003) explica que "es un procedimiento científico, un proceso sistemático de indagación, recolección, organización, análisis e interpretación de información o datos en torno a un determinado tema. Al igual que otros tipos de investigación, éste es conducente a la construcción de conocimientos. (Morales, 2003).

Del discernimiento del mismo autor se extrae que, la investigación documental "tiene la particularidad de utilizar como una fuente primaria de insumos, mas no la única y exclusiva, el documento escrito en sus diferentes formas" entre los que se encuentran los documentos impresos y electrónicos, tales como: libros enciclopedias, revistas, periódicos, diccionarios, monografías, tesis y otros documentos, correos electrónicos, CD Roms, bases de datos y páginas Web; y los recursos audiovisuales, que vendrían siendo: mapas, fotografías, ilustraciones, videos, programas de radio y de televisión, canciones, grabaciones y otros tipos de medios de información. Finalmente indica que: Como en el proceso de investigación documental se dispone, esencialmente, de documentos; que son el resultado de otras investigaciones, de reflexiones de teóricos, lo cual representa la base teórica del área objeto de investigación; el conocimiento se construye a partir de su lectura, análisis, reflexión e interpretación de dichos documentos. (Morales, 2003)

\section{Resultados.}

El VPH forma parte de una familia de virus que se trasmite a través del contacto sexual. El VPH puede afectar tanto a mujeres como a hombres. La mayoría de las personas estarán infectadas por el VPH alguna vez en la vida, pero sin consecuencias. El VPH puede ser transmitido incluso cuando una persona infectada no tenga signos ni síntomas. Las verrugas genitales ocasionadas por el virus VPH pueden desaparecer por sí mismas o pueden requerir tratamiento, pero no suelen causar cáncer. 
Noralma K. Jaime-Hernandez; Gerardo A. Granja-Carrión; Mario W. Pacheco-Moreira; Yuly S. Mitte-Baque; Ana M. Zambrano-Zambrano; Jhon A. González-Macas

Aunque no hay una cura para la infección por VPH, las verrugas, lesiones y cambios precancerosos y cancerosos causados por el virus pueden ser tratados. (OPS, 2019)

Aunque la infección es provocada por los mismos tipos de virus en el varón y la mujer, las lesiones difieren clínica e histológicamente. En la mujer: son característicamente blandas, de color gris o rosado, pedunculadas y suelen encontrarse en racimos. Las zonas más afectadas son la vulva, la pared vaginal, el cuello uterino (son causantes de cáncer), la piel de alrededor de la zona genital (periné) y el ano, mientras que, en el hombre, son igualmente blandas, en el pene suelen presentarse lisas y papulares, mientras que en las del perineo se asemejan a una coliflor. A menudo, aparecen primero en el prepucio (tejido que cubre el pene) y el glande, y luego en el escroto (piel externa de los testículos) y la región perianal. Otros casos suelen ser en la boca y en la garganta, lugares donde el VPH produce el papiloma oral y el papiloma laríngeo. También puede haber el papiloma de la conjuntiva del ojo y el papiloma nasal. (Crespo, 2008)

\section{Tipos de VPH}

De acuerdo con la Sociedad Americana Contra El Cáncer el sistema inmune del organismo humano, en la mayoría de los casos, es capaz de eliminar la infección por sí mismo, pero se dan las veces en que la infección no desaparece. Cuando la infección se vuelve crónica, o perdurable en el tiempo, especialmente la causada por determinadores tipos de VPH considerados de alto riesgo, puede provocar con el paso del tiempo el desarrollo de cierto tipo cáncer.

Aclaran que los VPH de bajo riesgo (BR / VPH-BR), llamados así porque "muy rara vez se convierten en cáncer", son aquellos que "pueden causar verrugas (papilomas) en o alrededor de los genitales y el ano tanto en hombres como en mujeres. Las mujeres también pueden presentar verrugas en el cuello uterino y la vagina"; mientras que por el contrario, aquellos que sí pueden causar cáncer tanto en hombres como en mujeres, por ende llamados de alto riesgo (AR / VPH-AR), son aquellos de tipo 16 y 18, por ejemplo, y son por los que "los médicos se preocupan más por los cambios celulares y los precánceres, ya que éstos tienden a transformarse en cánceres con el paso del tiempo". (SAC, 2017) 
Se han descrito más de 200 tipos de VPH, los cuales tienen tropismo por epitelios escamosos estratificados, infectando piel, mucosa oral y/o del tracto ano-genital. Los tipos de VPH cutáneos, entre ellos los tipos 1, 2, 3, 7 y 10, tienen como blanco principalmente manos y pies, formando verrugas típicas de la infección. Los VPH que tienen preferencia por tejidos mucosos infectan las células epiteliales basales de la boca, garganta tracto respiratorio o epitelio ano-genital y en cualquiera de ellos VPH podría dar origen a un proceso carcinogénico.

Los VPH transmitidos por vía sexual son aproximadamente 40, los que pueden ser agrupados en VPH de bajo riesgo (BR) y alto riesgo (AR) oncogénico. Los VPH de BR incluyen los tipos 6, 11, 42, 43 y 44, entre los más frecuentes. De ellos, los más importantes son los tipos 6 y 11, que producen verrugas genitales tanto en hombres como en mujeres. Los VPH de AR son los tipos 16, $18,31,33,34,35,39,45,51,52,56,58,59,66,68$ y 70. De estos tipos virales, el más importante y más frecuente en el cáncer de cuello uterino (CCU) es VPH 16. (Silva et al.; 2013; p. 186)

\section{Detección del VPH}

En la actualidad, el método más utilizado para la detección de VPH en el hombre es la amplificación de ácidos nucleicos utilizando la técnica de reacción de polimerasa en cadena (RPC) y sus diferentes variaciones. La alta sensibilidad de la técnica permite detectar hasta 3,9 copias del ADN viral al comienzo de la reacción. Por otro lado, la utilización de partidores de consenso que amplifican zonas de la región L1 del genoma viral, permiten la detección y posterior tipificación de los diferentes genotipos virales a partir de la amplificación del ADN de una única muestra. (Silva et al.; 2013; p. 188)

Debido a que la prueba de VPH es una tecnología nueva que aún no está disponible en todos los países, es muy importante que la mujer se realice un examen de detección temprana del cáncer cervicouterino, el PAP o citología, o la IVAA, para prevenir que lesiones precancerosas se transformen en cáncer. (OPS, 2019)

El Centro para el Control y la Prevención de Enfermedades (Center for Disease Control and Prevention / CDC, por sus siglas en inglés ente adscrito al Departamento de Salud y Servicios Humanos de los Estados Unidos (U.S. Department of Health \& Human Services / HHS, por sus siglas en inglés) exponen que: 
Noralma K. Jaime-Hernandez; Gerardo A. Granja-Carrión; Mario W. Pacheco-Moreira; Yuly S. Mitte-Baque; Ana M. Zambrano-Zambrano; Jhon A. González-Macas

Existen dos pruebas de detección que pueden ayudar a prevenir el cáncer de cuello uterino o a encontrarlo en etapas iniciales:

La prueba de Papanicoláu (o citología vaginal) busca precánceres, que son cambios en las células del cuello uterino que podrían convertirse en cáncer si no se tratan en forma adecuada. La prueba del VPH busca el virus (virus del papiloma humano) que puede causar este tipo de cambios celulares.

Ambas pruebas se pueden hacer en un consultorio o centro médico. Durante la prueba, el médico utiliza un instrumento de plástico o metal denominado espéculo para ensanchar la vagina. Esto permite que el médico examine la vagina y el cuello uterino, y recolecte algunas células y moco del cuello uterino y del área que lo rodea. Las células son enviadas a un laboratorio. Si le están haciendo una prueba de Papanicoláu, se revisarán las células para ver si se ven normales. Si le están haciendo la prueba del VPH, se le harán pruebas a las células para ver si tienen el VPH. (CDC, 2018)

\section{Otras Alternativas tecnológicas para la detección}

Inspección visual con ácido acético (IVA). La inspección visual con ácido acético (VIA) puede ser una alternativa a las pruebas citológicas o se puede usar junto con la prueba de Papanicolau o el ADN del VPH. VIA implica lavar el cérvix con ácido acético (vinagre) del 3\% al 5\% durante un minuto y luego observar el cérvix a simple vista. Si se observan áreas blancas bien definidas y características cerca de la zona de transformación, la prueba se considera positiva para los cambios de células precancerosas o cáncer invasivo temprano. VIA no requiere un laboratorio o capacitación intensiva del personal. Los resultados están disponibles de inmediato, lo que permite el tratamiento en una sola visita y, por lo tanto, reduce la pérdida para el seguimiento del paciente. La sensibilidad de VIA es tan buena o mejor que la prueba de Papanicolaou, pero al igual que la prueba de Papanicolaou, la inspección visual es subjetiva y se necesita supervisión para el control de calidad de los métodos de inspección visual. Una ventaja adicional de VIA no ofrecida por las pruebas de ADN de Pap o HPV es que permite a los proveedores identificar la pequeña proporción de lesiones positivas que no son adecuadas para el tratamiento con crioterapia, un modo de tratamiento apropiado para entornos de recursos limitados. Una implicación de esto es que, si la prueba de detección primaria se realiza mediante pruebas de Papanicolau, VIA o VPH, la decisión de no tratar con crioterapia solo se puede tomar con VIA (a menos que haya un colposcopio disponible). Por lo 
tanto, VIA se puede utilizar como prueba de detección primaria o para la clasificación del tratamiento posterior a la prueba de Papanicolau o VPH primaria.

Prueba del ADN del VPH. Las nuevas pruebas pueden detectar ADN de tipos de VPH que causan cáncer en frotis vaginal o cervical. Se extrae una muestra de células del cuello uterino o la vagina con un pequeño cepillo o hisopo, y la muestra se envía a un laboratorio para su procesamiento. Una ventaja de las pruebas de ADN del VPH es que cuando las condiciones son ideales, no es tan subjetivo como el examen visual y citológico. Una revisión de los estudios concluyó que las pruebas de ADN del VPH son particularmente valiosas para detectar lesiones precancerosas de alto grado en mujeres mayores de 30 años. Es probable que las infecciones por VPH en mujeres menores de 30 años se curen por sí mismas, por lo que las pruebas a mujeres de esa edad conducirían a frecuentes tratamientos excesivos. (RHO, 2012)

\section{Factores influyentes}

Durante la pubertad y el embarazo, la zona del ectocérvix se amplía, lo cual facilita la exposición al VPH y, por tanto, la infección. Esta situación explica por qué el inicio de la vida sexual activa y el primer embarazo a edades tempranas, así como la multiparidad, son de los factores de riesgo más fuertemente asociados al desarrollo de cáncer cervicouterino, además del hecho de tener múltiples parejas que a su vez tienen parejas con múltiples parejas. (Ochoa, Guarneros, \& Velasco, 2015)

La infección persistente (más de 12 meses) por alguno de los 15 tipos oncogénicos es condición necesaria pero no suficiente para la aparición de displasia y cáncer de cuello de útero. Múltiples estudios han analizado la influencia de otros factores, entre estos:

- Multiparidad (cinco o más alumbramientos).

- Uso de terapia hormonal más de 5 años.

- Mantener sexo con hombre que haya tenido seis o más parejas sexuales.

- Coinfección por C. trachomatis y virus herpes simple tipo 2 (VHS-2).

- Nivel socioeconómico bajo.

- Déficit de micronutrientes.

- Tabaquismo.

- Comienzo de relaciones sexuales a edades tempranas. 
Noralma K. Jaime-Hernandez; Gerardo A. Granja-Carrión; Mario W. Pacheco-Moreira; Yuly S. Mitte-Baque; Ana M. Zambrano-Zambrano; Jhon A. González-Macas

- No utilizar preservativos.

- Pertenecer a algunos grupos sociales marginales (prostitutas, adictas a drogas o a alcohol, reclusas y portadoras del virus de la inmunodeficiencia humana [VIH]). (Gavilán \& Padilla, 2013)

\section{Vacunas frente al VPH}

Tomando como referencia la publicación de Organización Panamericana de la Salud (OPS / PAHO, por sus siglas en inglés) de 2010, puede decirse que la implementación de vacunas contra el VPH viene ejecutándose desde hace tiempo, y entre estas:

[...] se incluyen una vacuna tetravalente que contiene los genotipos $6,11,16$ y 18 y otra bivalente que contiene los genotipos 16 y 18. En los ensayos clínicos, se ha comprobado que ambas vacunas son muy seguras y tienen gran inmunogenia y una eficacia superior a $90 \%$ en la prevención de la infección y las lesiones precancerosas causadas por los tipos 16 y 18 del VPH cuando se administran a las adolescentes antes de su iniciación sexual. Se ha autorizado su uso en mujeres de 9 a 26 años, sobre la base de los datos obtenidos de ensayos de la eficacia y la inmunogenia. Se ha comprobado que las vacunas confieren protección durante cinco años como mínimo (el período de seguimiento más largo sobre el cual se han publicado datos) y tal vez durante mucho más tiempo. Se planea realizar estudios de seguimiento de 14 años como mínimo para determinar la duración de la protección. Además, todavía se están recopilando datos clínicos sobre la eficacia de la vacuna contra el VPH en los niños varones.

Veintiocho países de las Américas han autorizado la vacuna contra el VPH y en Canadá y los Estados Unidos actualmente se está administrando la vacuna en programas de inmunización; en Costa Rica, México y Perú se está ensayando la vacuna contra el VPH en proyectos de demostración o investigaciones. La asequibilidad de las vacunas contra el VPH y los preparativos necesarios para introducirlas como parte de un programa integral de control del cáncer cervicouterino siguen siendo un problema para los programas de salud pública. Las actuales vacunas contra el VPH han sido precalificadas por la OMS como vacunas nuevas, lo cual permite a los países en desarrollo realizar compras a través de organismos de las Naciones Unidas y del fondo rotatorio de la OPS. 
Las vacunas contra el VPH no son un sustituto de la educación sanitaria y el tamizaje. Un programa integral para combatir el cáncer cervicouterino deberá incluir todos los componentes: la educación sanitaria, el tamizaje, el diagnóstico, el tratamiento y los cuidados paliativos, aun después de que se puedan introducir las vacunas según la asequibilidad, la sostenibilidad y todos los preparativos necesarios para la introducción de vacunas nuevas (por ejemplo, la capacitación de los proveedores, el fortalecimiento de la cadena de frío y de los laboratorios, etc.) (PAHO, 2010)

Específicamente, las vacunas a las que se hace referencia son Gardasil@ y GlaxoSmithKline, Cervarix ${ }^{\circledR}$ aprobadas y comercializadas desde 2006 bajo la licencia de Merck \& Co. Inc.'s, estando autorizadas en más de 100 países en todo el mundo desde entonces hasta la actualidad. (RHO, 2012)

\section{Prevención del VPH}

Del trabajo de Luna \& Sánchez (2014) logra extraerse que, en la identificación de la infección por VPH un elemento clave es el enfoque de la prevención.

Tanto el concepto como la práctica de la prevención difieren entre la población atendida y el sector médico, que trabaja según los lineamientos de la biomedicina. Analizar estas diferencias conceptuales y prácticas en torno a la prevención resulta un elemento clave, ya que permite una adecuada identificación de acciones efectivas para disminuir la infección por VPH [...]. (Luna \& Sánchez, 2014)

Las autoras se apoyan en la idea de (Sebastián, 2001) para referirse a la prevención primaria, siendo éste quien dijo "se enfoca en evitar la aparición de la enfermedad utilizando medidas que disminuyan los factores de riesgo y favoreciendo comportamientos y hábitos más saludables entre la población" (p. 184-185), agregando las tratadistas que eso pudiera ser "el uso de preservativos o el mantenimiento de una relación monógama, lo cual disminuiría considerablemente la exposición a la infección”. Continúan basándose en la misma referencia bibliográfica para referirse a la prevención secundaria del VPH, y al respecto citan que "[...] tiene como objetivo reducir la mortalidad y la morbilidad mediante un diagnóstico precoz que incluya aspectos relacionados con el tratamiento" (Sebastián; 2001; p. 184-185). En este recurso se ubican los programas de detección oportuna, las campañas publicitarias que brindan información a la población y la respuesta eficaz de las instituciones ante diagnósticos de lesiones cervicouterinas, en todo lo cual es determinante la 
Noralma K. Jaime-Hernandez; Gerardo A. Granja-Carrión; Mario W. Pacheco-Moreira; Yuly S. Mitte-Baque; Ana M. Zambrano-Zambrano; Jhon A. González-Macas

comunicación entre el personal médico y las mujeres que requieren atención. (Luna \& Sánchez, 2014)

Es conveniente educar a niñas y niños en sexualidad y la infección por el VPH, de acuerdo a su edad y su cultura; así como promover el uso de la vacuna contra el VPH y proveerla en escuelas y centros de salud, como también promover el uso y proveer de condones para aquellos que han iniciado su actividad sexual; y advertir a niños y niñas sobre el uso de tabaco pues es un factor de riesgo para todos los tipos de cáncer, incluyendo el cáncer cervicouterino. El uso de condones no provee protección completa contra el VPH, aunque puede proteger de otras infecciones y de embarazos no deseados. Las medidas de limpieza personal no ayudan a prevenir la infección por el VPH. (OPS, 2019)

\section{Consideraciones preventivas para la detección del VPH}

Inicialmente se debe reconocer que las consideraciones preventivas para la detección del VPH pueden ser asumidas desde dos puntos de vista. El primero, estaría referido a las consideraciones que dependen o pueden asumir, tanto el individuo (primordialmente, porque sería el posible infectado y es quien eventualmente buscará aclara sus dudas) como el médico, o bien el consenso de ambos. Esto es porque, por una parte, el paciente instruido (de manera individual) y por la otra, el médico tratante, individualmente o en conjunto, bien pudieran responder a la pregunta: ¿Qué o cuáles consideraciones previas son la que deben observarse antes de la detección del VPH? Las respuestas alcanzadas eventualmente por el paciente, es probable que estriben entre consideraciones psicoemocionales por el posible contagio e ineludiblemente con la decisión de cómo o cuál método de detección (y/o diagnóstico) de VPH escogerá, bien sea que se aplique un determinado método en base a una recomendación médica o que particularmente evalúe las opciones disponibles o que se ofrecen en el mercado sin prescripción facultativa. Por otra parte, estarían las consideraciones preventivas que al respecto observaría un médico, y estas seguramente oscilarían entre aquellas que tengan una justificación clínico-científicas que surgen de su especial conocimiento, refiriendo así cuál es la prueba correspondiente y conteste a la sensibilidad y la especificidad requerida.

Independientemente de que un hombre o mujer que se instruya sobre esta enfermedad, inicialmente tenga la posibilidad de detectar física o sintomatológicamente el VPH, hay que tener muy claro que cualquier de éstos, ante la duda, requerirán y necesitarán de la certeza por el contagio o no con dicha 
infección, entonces, se considera que lógicamente la manera más idónea, responsable e inequívoca de verificarlo es a través del sometimiento a una de las pruebas de VPH, quedando la escogencia de cualquiera de éstas (entre las clínicamente más ortodoxas y las más novedosas), básicamente a criterio consensuado entre el interesado y su especialista, quienes en conjunto tendrán en consideración otros factores particulares e ineludibles.

Lo antes dicho sirve de preámbulo para ahora exponer sobre el otro punto de vista desde el cual pueden asumirse las consideraciones preventivas para la detección del VPH, y ese no es más que el punto de vista gubernamental; aspecto que responde al verdadero enfoque que debe exponer sobre este aspecto.

Lo que aquí se quiere destacar es la importancia de la selección de la prueba a aplicar para la detección del VPH, pues con estas desde siempre se ha buscado que cada vez más alcancen un mayor nivel de sensibilidad y especificidad. Ello se ha dejado ver claramente en un valioso aporte que al respecto a desarrollado la OPS (PAHO), organismo que, tras su constante labor a nivel internacional, ejecutada sistemática y responsablemente a lo largo de tantos años desde su creación, en la que siempre considerando las particularidades sociopolíticas, económicas, culturales, geográficas y otras de cada región (país), ha recabado la información pertinente para precisamente describir cuales deberían ser las consideraciones previas a la selección de una prueba de VPH, y estas son:

- ¿Qué tipos de VPH se detectan con la prueba?

- ¿Cómo se presentan los resultados: positivo- negativo o por tipo de VPH?

- ¿Cuáles son los requerimientos y costos de los insumos y equipo?

- ¿Cuál es el tamaño adecuado del lote para procesar las muestras?

- ¿Cuánto tiempo toma el procesamiento de la muestra?

- ¿Qué tipo de capacitación se requiere para procesar la prueba?

- ¿Qué apoyo está disponible en el país para la instalación y el mantenimiento del equipo?

- ¿Cómo se controla la calidad de los resultados de la prueba?

- ¿Se puede utilizar la prueba de VPH en forma de autotoma?

- ¿Cuáles son los requisitos para el almacenamiento, caducidad y otros aspectos de la gestión de la cadena de abastecimiento? 
Noralma K. Jaime-Hernandez; Gerardo A. Granja-Carrión; Mario W. Pacheco-Moreira; Yuly S. Mitte-Baque; Ana M. Zambrano-Zambrano; Jhon A. González-Macas

- ¿Cuáles son las condiciones o arreglos con los distribuidores locales para la provisión de la prueba, incluyendo el precio?

- ¿Qué aspectos o insumos están incluidos en el precio (reactivos, equipos, pipetas, etc.)?

- Antes de seleccionar una prueba de VPH entre la amplia variedad disponible en el mercado, se debe realizar un análisis de costo-beneficio y considerar la factibilidad de implementar la prueba en el contexto del programa de tamizaje.

- Se deben elegir solo las pruebas de VPH que tienen una validación clínica.

- Las pruebas autorizadas por agencias reguladoras, tal como la FDA, serían opciones seguras.

- Introducir una prueba en un programa y luego cambiarla por otra será difícil y tendrá implicaciones de costos.

- Las pruebas tienen fecha límite, por ejemplo 9 meses o 12 meses, y hay que tomar en cuenta los aspectos de la gestión de la cadena de abastecimiento al elegir la prueba y su aplicación en el programa. (PAHO, 2016)

\section{Conclusión.}

Sigue siendo especialmente trascendental que, mediante programas se siga brindando información a la población en general, y particularmente a los adolescentes, tanto sobre la prevención de la infección por el VPH como de las otras infecciones de transmisión sexual y comportamientos sexuales sanos, no obstante, tanto para aquellos países o gobiernos que mediante sus políticas ya han acogido oficialmente alguna prueba para la detección del VPH como para aquellos que aún están en ese proceso, a tales fines sigue siendo recomendable que tengan en consideración la validación clínica de la prueba, aspectos operacionales y logísticos, además de los costos y beneficios de la prueba.

\section{Bibliografia.}

American Chemical Society. (2018). VPH y cáncer. Washington, United States: ACS.

CDC. (24 de 08 de 2018). ¿Qué debo saber sobre la detección? Obtenido de Centers for Disease Control and

Prevention: https://www.cdc.gov/spanish/cancer/cervical/basic_info/screening.htm?CDC_AA_refVal=ht tps $\% 3 \mathrm{~A} \% 2 \mathrm{~F} \% 2 \mathrm{Fwww} . c d c . g o v \% 2 \mathrm{Fstd} \% 2 \mathrm{Fspanish} \% 2 \mathrm{Fvph} \% 2 \mathrm{Fhpv}-\mathrm{pap}$-sp.htm 
Crespo, F. (17 de 12 de 2008). intramed.net. Obtenido de intramed.net: https://www.intramed.net/contenidover.asp?contenidoID $=50284$

Gavilán, E., \& Padilla, J. (2013). AMF, 9(4). Obtenido de http://amfsemfyc.com/web/article_ver.php?id=1130

Luna, M., \& Sánchez, G. (2014). Posibilidades sociales de prevención de la infección por virus del papiloma humano y de cáncer cervicouterino en San Cristóbal de Las Casas, Chiapas, México. LiminaR, 12(2), 67-80. Obtenido de http://www.scielo.org.mx/scielo.php?script=sci_arttext\&pid=S1665-80272014000200005

Morales, O. (2003). Fundamentos de la Investigación Documental y la Monografía. En F. d. Grupo Multidisciplinario de Investigación en Odontología, \& N. E. Rincón (Ed.), Manual para la elaboración y presentación de la monografía (pág. 20). Merida, Merida, Venezuela: Universidad de Los Andes (ULA). Obtenido de Www.saber.ula.ve/bitstream/handle/123456789/16490/fundamentos_investigacion.pdf

Ochoa, F., Guarneros, D., \& Velasco, M. (Mayo-Junio de 2015). Infección por virus del papiloma humano en mujeres y su prevención. Gaceta Mexicana de Oncología, 14(3), 133-192.

OPS. (2019). El Virus del Papiloma Humano y el Cáncer Cervicouterino. Organización Panamericana de la Salud. Obtenido de https://www.paho.org/hq/index.php?option=com_docman\&view=download\&category_slug =hojas-informativas-3577\&alias=32863-hoja-informativa-3-vacuna-conra-vph$863 \&$ Itemid $=270 \&$ lang $=\mathrm{es}$

OPS. (2019). El Virus del Papiloma Humano y el Cáncer Cervicouterino. Organización $\begin{array}{lllll}\text { Panamericana de la } & \text { Salud. } & \text { Obtenido }\end{array}$ https://www.paho.org/hq/index.php?option=com_docman\&view=download\&category_slug =hojas-informativas-3577\&alias=32862-hoja-informativa-2-virus-papiloma-humano-vph$862 \&$ Itemid $=270 \&$ lang $=\mathrm{es}$

PAHO. (2010). rho.org. Obtenido de rho.org: http://www.rho.org/spanish.htm

PAHO. (02 de 09 de 2018). Cáncer cervicouterino. Obtenido de www.paho.org: https://www.paho.org/hq/index.php?option=com_topics\&view=article\&id=348\&Itemid=409 $36 \&$ lang $=\mathrm{es}$

RHO. (2012). rho.org. (P. f. (PATH), Editor) Obtenido de rho.org: http://www.rho.org/aboutcervical-cancer.htm

RHO. (2012). rho.org. Obtenido de rho.org: http://www.rho.org/about-cervical-cancer.htm

Rivera, A. (2018). Experiencia de estudios realizados sobre Papiloma Virus Humano (VPH) en diferentes provincias del Ecuador. Revista cientifica digital INSPILIP, 4(2). doi:DOI: 10.31790/inspilip.v2i1.39.g44

Silva, C., Waliszewski, S., Lozoya, H., \& Carrillo, M. (Julio-Diciembre de 2013). Factores relacionados con la infección del virus de papiloma humano en mujeres. Revista de Investigación Científica en Ciencias de la Salud, 8(2), 39-45. 
Noralma K. Jaime-Hernandez; Gerardo A. Granja-Carrión; Mario W. Pacheco-Moreira; Yuly S. Mitte-Baque; Ana M. Zambrano-Zambrano; Jhon A. González-Macas

Silva, R., León, D., Brebi, P., Ili, C., Roa, J., \& Sánchez, R. (2013). Diagnóstico de la infección por virus papiloma humano en el Hombre. Revista Chilena Infectología, 30(2), 186-192. Obtenido de https://scielo.conicyt.cl/pdf/rci/v30n2/art09.pdf 